\title{
Circulating Immune Complexes and Immunoglobulin A Rheumatoid Factor in Patients with Mesangial Immunoglobulin A Nephropathies
}

\author{
Cecil Czerkinsky, William J. Koopman, Susan Jackson, Jennifer E. Collins, Sylvia S. Crago, Ralph E. Schrohenloher, \\ Bruce A. Julian, John H. Galla, and Jiri Mestecky \\ Departments of Microbiology and Medicine, University of Alabama at Birmingham and Veterans Administration Medical Center, \\ Birmingham, Alabama 35294
}

\begin{abstract}
Circulating immune complexes (CIC) containing IgA and $\mathrm{C} 3$ were elevated in $48 \%$ of IgA nephropathy patients; IgA1 was the predominant subclass. IgA1-IgG CIC were detected in 44\%, IgA2IgG CIC in 7\%, and IgM-IgA1 CIC in 16\% of the patients. No IgM-IgA2 CIC were detectable. Sucrose gradient ultracentrifugation indicated that IgG-IgA1 CIC were predominantly of intermediate (13-19S) size whereas IgA1-C3 CIC sedimented from $11 S$ to $19 \mathrm{~S}$. At acid $\mathrm{pH}$, isolated $\mathrm{CIC}$ revealed the presence of substantial amounts of 7S IgA. One third of the patients had elevated serum IgA rheumatoid factor (RF) of both polymeric and monomeric forms despite normal levels of IgM-RF; $87 \%$ of patients with elevated IgA-RF had IgA1-IgG CIC. These results indicate that the IgA1 component of CIC in patients with IgA nephropathy is not necessarily of mucosal origin and suggest that a portion of these CIC consists of IgA RF immunologically complexed with autologous IgG.
\end{abstract}

\section{Introduction}

IgA nephropathies (IgA NP) ${ }^{1}$ represent one of the most common groups of glomerulonephritides in humans (1). Diffuse mesangial localization of IgA in kidney biopsy specimens from patients with IgA NP is the most distinctive feature of the disease. Nonetheless, it has now become clear that, in addition to idiopathic IgA NP (Berger's disease), several other diseases, such as HenochSchoenlein purpura, celiac disease, alcoholic liver cirrhosis, and dermatitis herpetiformis share this common immunopathologic feature (2-10).

A putative nephritogenic role has been ascribed to IgA-containing circulating immune complexes (CIC) in mesangial IgA NP (11). This concept has arisen from early immunohistologic studies performed on kidney biopsy specimens of IgA NP patients showing mesangial deposits of IgA with a granular pattern, similar to that observed in other glomerulonephritides in which $\mathrm{CIC}$ are thought to play a major inflammatory role (12). The

Address reprint requests to Dr. Mestecky, Department of Microbiology, University of Alabama at Birmingham, Birmingham, AL 35294.

Received for publication 10 December 1985 and in revised form 27 January 1986

1. Abbreviations used in this paper: $\mathrm{CIC}$, circulating immune complexes; ELISA, enzyme-linked immunosorbent assay; FBS, fetal bovine serum; IgA NP, IgA nephropathy; PAP, horseradish peroxidase IgG antiperoxidase antibody complexes; PEG, polyethylene glycol; RF, rheumatoid factor; RIA, radioimmunoassay; SDU, standard deviation units.

J. Clin. Invest.

(c) The American Society for Clinical Investigation, Inc.

0021-9738/86/06/1931/08 $\$ 1.00$

Volume 77, June 1986, 1931-1938 frequent presence of complement components in these deposits (1), the inability to demonstrate convincingly antimesangial antibodies in the sera of IgA NP patients (7), and the recurrence of IgA nephropathy in renal allografts (13) are compatible with the view that IgA-containing CIC are deposited in the glomeruli. This hypothesis has gained further support from recent studies demonstrating elevated levels of CIC that contain IgA not only in patients with primary IgA NP (14-20), but also in patients with Henoch-Schoenlein purpura (16, 21-23), dermatitis herpetiformis $(23,24)$, and IgA NP associated with liver cirrhosis $(25,26)$. In rodents, IgA-containing CIC either infused $(27)$ or induced by enteral (28) or parenteral (29) immunization with defined antigens can deposit in the mesangium and initiate a proliferative glomerulonephritis similar to that observed in humans. Despite this, attempts to isolate a specific IgA-CIC-associated antigen in humans have been unsuccessful and the pathogenic role of these $\mathrm{CIC}$ remains elusive.

Initial immunofluorescence studies with polyclonal antisera suggested that IgA2 was the predominant subclass of IgA in glomerular deposits of patients with mesangial IgA NP (30). In contrast, more recent studies with subclass-specific monoclonal antibodies have indicated that IgAl is the predominant, often exclusive, subclass present in these deposits (18, 31-34). In addition to the absence of detectable secretory component $(15,35$, 36 ), these observations suggest that the $\operatorname{IgA}$ deposited may not be of secretory origin. Less information (26) is available regarding the properties of IgA present in CIC-like material detected in patients with mesangial IgA NP. Such information could provide clues as to the origin, be it mucosal or systemic, of the $\operatorname{IgA}$ component of these CIC.

In addition to IgA-CIC, CIC that contain IgG have been detected in patients with IgA NP $(16,19,20)$, Henoch-Schoenlein purpura $(16,19,21,22)$ and alcoholic liver cirrhosis $(25$, $37,38)$. Although conflicting results were reported concerning the frequency of $\operatorname{IgG}-\mathrm{CIC}(18,22,37,38)$, the pathogenic relevance of these $\mathrm{CIC}$ has not yet been adequately explained.

The occurrence of IgA- and IgG-CIC, as well as the frequent co-deposition of IgA and IgG in glomerular mesangia have prompted us to examine the possible occurrence of CIC that contain both IgG and IgA in IgA NP. We now report that sera from a substantial proportion of such patients exhibit increased levels of CIC containing both IgG and IgA I. In addition, IgA 1 rheumatoid factor (RF) activity was demonstrated in most patients with IgG-IgA 1-CIC in the absence of elevated serum IgM RF, suggesting that at least a portion of these CIC consists of IgA1 RF immunologically complexed with autologous IgG.

\section{Methods}

Patients and controls. Studies were carried out in 32 patients with IgA NP. The diagnosis of idiopathic IgA nephropathy (Berger's disease) was 
established in 28 patients by the demonstration of mesangial deposition of IgA as the dominant or codominant immunoglobulin isotype (34) and the absence of the manifestations of other systemic diseases. The diagnosis of Henoch-Schoenlein purpura was established in one patient in whom the above immunofluorescence findings of IgA NP were accompanied by abdominal pain, joint pain, and purpura. Of the remaining three patients, one had dermatitis herpetiformis, one had had a previous kidney biopsy with diagnosis of focal glomerulosclerosis and one had coincidental diabetes mellitus. 21 of the patients were white males, one was a black male, and the remainder were white females; this distribution is in keeping with previously reported demographic data $(39,40)$. Ages ranged from 9 to $82 \mathrm{yr}$ (median $26 \mathrm{yr}$ ). Serum creatinine concentrations ranged from 0.5 to $15.2 \mathrm{mg} / \mathrm{dl}$ (median $1.0 \mathrm{mg} / \mathrm{dl}$ ). Two patients had received kidney transplants which function normally. The 24-h urinary protein excretion ranged from 0 to $4.9 \mathrm{~g}$ (median 0.7 ) and the magnitude of hematuria from 0 to 1,000 red blood cells per high power field (median 5).

21 healthy laboratory volunteers (median age $31 \mathrm{yr}$, ranging from 17 to $53 \mathrm{yr}$ ) were utilized as controls.

CIC assays. Freshly separated serum samples were precipitated with polyethylene glycol (PEG) (PEG 6000, Fisher Scientific, Orangeburg, NY) using the conditions detailed by Kauffman et al. (41) to prepare fractions enriched for CIC. Before analysis, each PEG precipitate was

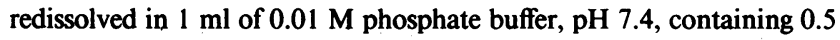
$\mathrm{M} \mathrm{NaCl}, 0.05 \%$ Tween 20 and $5 \%$ fetal bovine serum (FBS, Irvine Scientific, Santa Ana, CA) (CIC buffer).

Levels of CIC containing C3 and IgA, IgG, or IgM were estimated by a modification (42) of the solid-phase anti-C3 assay (43). Individual wells of microtiter plates (Linbro, Flow Laboratories, Inc., McLean, VA) were coated overnight with $F\left(a b^{\prime}\right)_{2}$ fragments of goat anti-human $C 3$ (Cooper Diagnostics, West Chester, PA) diluted to $10 \mu \mathrm{g} / \mathrm{ml}$ in $0.01 \mathrm{M}$ phosphate-buffered saline (PBS), $\mathrm{pH}$ 7. The anti-C3 reagent was absorbed on insolubilized mouse immunoglobulins and affinity purified by chromatography on insolubilized human C3 (donated by Dr. J. Volanakis, University of Alabama at Birmingham). $100 \mu$ laliquots of redissolved PEG precipitates were added to anti-C3-coated wells at a dilution equivalent to $1: 100$ of the original serum volume. Plates were incubated at $37^{\circ} \mathrm{C}$ for $4 \mathrm{~h}$, washed with PBS containing $0.05 \%$ Tween 20 (PBS-Tween), and subsequently exposed to biotinylated $\mathrm{F}\left(\mathrm{ab}^{\prime}\right)_{2}$ fragments of goat antihuman IgA, IgM, or IgG (Tago, Inc., Burlingame, CA) appropriately diluted in PBS- $0.05 \%$ Tween-5\% FBS. The specificity of these reagents was established as previously described $(44,45)$. After overnight incubation at $4^{\circ} \mathrm{C}$, the plates were washed and subsequently incubated for $60 \mathrm{~min}$ with horseradish peroxidase-conjugated avidin (Sigma Chemical Co., St. Louis, MO) diluted to $0.5 \mu \mathrm{g} / \mathrm{ml}$ in PBS-Tween-FBS. After washing, the plates were exposed to enzyme substrate consisting of 2,2 '-azinodi(3-ethyl benzthiazoline sulfonic acid) (Sigma Chemical Co.) at a con-

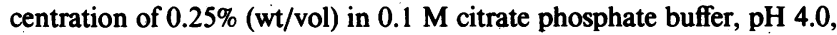
containing $0.0075 \% \mathrm{H}_{2} \mathrm{O}_{2}$. Color development was monitored spectrophotometrically at $414 \mathrm{~nm} 30$ min after addition of substrate using an automated reader (Titertek Multiskan, Flow Laboratories).

Levels of CIC containing $\mathrm{C} 3$ and either IgA1 or IgA2 were determined essentially as described above except that monoclonal antibodies to human IgA1 or IgA2 subclass (46) were substituted for the polyclonal antihuman immunoglobulin reagents. All reagents were used at concentrations determined to be optimal in preliminary checkerboard titration experiments. The washed plates were then incubated for $2 \mathrm{~h}$ at $37^{\circ} \mathrm{C}$ with biotinylated $\mathrm{F}\left(\mathrm{ab}^{\prime}\right)_{2}$ fragments of affinity-purified, goat anti-mouse IgG antibodies (Calbiochem-Behring Corp., San Diego, CA) previously absorbed against human serum. Bound antiglobulins were detected as above by stepwise addition of avidin-peroxidase and enzyme substrate.

Levels of CIC containing both IgG and IgA1 or IgA2 were determined by a solid-phase radioimmunoassay (RIA) using class- and subclass-specific monoclonal antiglobulins. Triplicate $100-\mu 1$ aliquots of redissolved PEG precipitates diluted 1:100 in CIC buffer were incubated for $4 \mathrm{~h}$ at $37^{\circ} \mathrm{C}$ in polystyrene Removawells (Immulon type II, Dynatech, Alexandria, VA) coated with monoclonal anti-human IgG (47). CIC containing both IgM and IgA1 or IgA2 were detected with monoclonal (IgG1, к) mouse anti-human IgM (47) antibodies that were substituted for the anti-IgG reagent. Wells coated with mouse monoclonal IgG1, $k$ of irrelevant specificity were used for control purposes. After washing with PBS, ${ }^{125}$ I-labeled monoclonal antibodies $(100,000 \mathrm{cpm}$ in $100 \mu \mathrm{l})$ to either IgA1 or IgA2 subclass were added. These reagents were radiolabeled by the lactoperoxidase method (48) using $10 \mu \mathrm{g}$ of protein and $0.25 \mathrm{mCi}$ of $\mathrm{Na}^{125} \mathrm{I}$ (Amersham Corp., Arlington Heights, IL). After incubation the plates were washed and bound radioactivity was measured in a gamma counter (Beckman Instruments, Inc., Palo Alto, CA). Background binding never exceeded $10 \%$ of the counts observed in antiglobulin coated wells.

Results of the different CIC assays are expressed in standard deviation units (SDU) according to the formula: $\mathrm{SDU}=(X \mathrm{t}-X \mathrm{n}) / \mathrm{SD}_{\mathrm{n}}$, where $X \mathrm{t}$ represents the mean value (counts per minute or absorbance) of each test sample, $X \mathbf{n}$ the mean value of a basic set of samples from at least 12 healthy blood donors assayed simultaneously, and $\mathrm{SD}_{\mathrm{n}}$ the standard deviation of the mean value obtained for the panel of healthy donors. Results were considered positive when the mean value of a test sample run in duplicate or triplicate exceeded that of the basic set of normal donors by at least 3 SDU.

$R F$ assays. Serum IgA- and IgM-RF were estimated by two different procedures. First, we used a solid-phase RIA (46) with polyclonal human IgG as solid-phase and either ${ }^{125}$ I-labeled affinity purified goat anti-human IgA (49) or anti-human IgM (50) antibodies as indicator reagents to detect bound RF. In addition, ${ }^{125}$ I-labeled mouse monoclonal anti-human IgA 1 or IgA2 antibodies were used to detect bound RF (49). Wells coated with bovine serum albumin (Sigma Chemical Co.) were used for control purposes. In some experiments, wells were coated with $F c$ and $F\left(a^{\prime}\right)_{2}$ fragments of human IgG to examine the specificity of the anti-IgG antibodies detected (50).

Additionally, we used a modification of a solid-phase enzyme-linked immunosorbent assay (ELISA) (42) for detection of RF. Affinity-purified $\mathbf{F}\left(\mathrm{ab}^{\prime}\right)_{2}$ fragments of rabbit anti-human IgA or IgM (Pelfreeze, Rogers $\mathrm{AZ}$ ) were used to capture immunoglobulins of the relevant isotype from serum samples. In order to detect RF activity in the bound immunoglobulins, soluble immune complexes consisting of horseradish peroxidase and rabbit IgG antiperoxidase antibodies (PAP) (Dakopats, Copenhagen, Denmark) were incubated on the plate. After washing and addition of enzyme substrate, the concentration of PAP bound to immobilized IgA or IgM RF was determined colorimetrically. IgG-RF levels were also examined by this procedure using a coating of affinity-purified $\mathrm{F}\left(\mathrm{ab}^{\prime}\right)_{2}$ fragments of rabbit anti-human IgG (Jackson Laboratories, Inc., Avondale, PA). This technique will be referred to as ELISA-PAP. Results of the different RF assays are expressed in SDU as described above for the CIC assays.

Sedimentation properties of CIC. Samples $(0.3 \mathrm{ml})$ of freshly redissolved PEG precipitates were applied onto 4.7-ml linear gradients of 12$30 \%(\mathrm{wt} / \mathrm{vol})$ sucrose preformed in 0.01 M PBS, $\mathrm{pH}$ 7.4. After ultracentrifugation at $37,500 \mathrm{rpm}$ at $4^{\circ} \mathrm{C}$ for $16 \mathrm{~h}$ in a Beckman L2 ultracentrifuge using a SW 50.1 rotor, fractions $(0.25 \mathrm{ml})$ were collected from the top. Prior to analysis for IgG-IgA1- or C3-IgA1-containing CIC, fractions were diluted (1:4 to 1:10) in CIC buffer. Standards consisted of purified IgG and IgM paraproteins.

Characterization of IgA molecular forms in isolated CIC. Redissolved PEG precipitates equivalent to $2 \mathrm{ml}$ of serum from three patients with high levels of IgA-CIC were incubated for $4 \mathrm{~h}$ at $37^{\circ} \mathrm{C}$ with $1 \mathrm{ml}$ of packed cyanogen bromide (CNBr)-activated Sepharose 4B beads (Pharmacia Fine Chemicals, Piscataway, NJ) to which $F\left(a b^{\prime}\right)_{2}$ fragments of anti-human $\mathrm{C} 3$ or anti-human IgG Fc had been covalently coupled. Bound material was eluted with $0.1 \mathrm{M}$ sodium acetate buffer, $\mathrm{pH} 3$, and concentrated to the original volume by centrifugation using Centricon microconcentrators YM 10 (Amicon Corp., Danvers, MA) and applied onto linear sucrose gradients in $0.1 \mathrm{M}$ acetate buffer, $\mathrm{pH} \mathrm{4}$, and ultracentrifuged as described above. Collected fractions $(250 \mu \mathrm{l})$ were neutralized with $30 \mu \mathrm{l}$ of $\mathrm{Na}_{2} \mathrm{HPO}_{4}$ to a final pH of 7.5 prior to analysis for IgA content by RIA as described elsewhere (44).

Sedimentation properties of $I g A R F$ in serum and isolated $C I C$. Serum samples $(0.3 \mathrm{ml})$, diluted $1: 2$ to $1: 10$ in PBS containing 5\% FBS, were ultracentrifuged on sucrose gradients, and the fractions were analyzed 
for the presence of polymeric and monomeric forms of IgA RF by solidphase RIA (51). Parallel analyses were carried out under dissociating conditions by ultracentrifugation on linear sucrose gradients in $0.1 \mathrm{M}$ sodium acetate buffer, $\mathrm{pH} 4$.

IgG-IgA1 CIC were isolated from redissolved PEG precipitates equivalent to $2 \mathrm{ml}$ of serum from five patients with high levels of serum IgA RF. After dissociation and sucrose gradient ultracentrifugation at acid $\mathrm{pH}$, the fractions were collected, neutralized and assayed for IgA RF activity.

Serum levels of immunoglobulins. Serum levels of IgA, IgM, and IgG were measured by radial immunodiffusion. Concentrations of IgA ranged from 61 to $1,247 \mathrm{mg} / \mathrm{dl}$ (median $298 \mathrm{mg} / \mathrm{dl}$ ), IgM from 45 to $623 \mathrm{mg} /$ dl (median $123 \mathrm{mg} / \mathrm{dl}$ ), and IgG from 191 to $2,575 \mathrm{mg} / \mathrm{dl}$ (median 1,060 $\mathrm{mg} / \mathrm{dl})$.

Statistical analyses. Differences between groups were determined by the Mann-Whitney rank sum test. Associations between the variables studied were determined by the Spearman rank-order correlation test.

\section{Results}

Circulating immune complexes. The results of the assays for $\mathrm{C} 3-$ containing CIC are summarized in Fig. 1. CIC containing C3 and $\operatorname{IgA}$ were elevated in $48 \%(12 / 25)$ of the patients studied. With subclass-specific monoclonal anti-IgA reagents, $44 \%$ $(11 / 25)$ of the patients had significantly higher levels of IgA1C3-containing CIC as compared with healthy volunteers ( $P$ $<0.001)$. In contrast, none of 28 patients examined had evidence of IgA2-C3-containing CIC. Levels of IgA1-C3 CIC correlated with the magnitude of proteinuria $(r=0.44 ; P=0.045)$ but not with serum creatinine concentrations $(P>0.5)$ or with the magnitude of hematuria $(P>0.5)$. IgG-C3-containing $\mathrm{CIC}$ were detected in $24 \%(5 / 21)$ of the patients studied and correlated with levels of CIC containing $\operatorname{IgA} 1-\mathrm{C} 3(r=0.72, P=0.0003)$. IgM-C3-containing CIC were elevated in only $9 \%(2 / 21)$ of the patients tested.

CIC containing both IgG and IgAl were found significantly increased in $46 \%(12 / 30)$ of IgA NP patients as compared to a panel of sera from 14 healthy blood donors $(P<0.005)$ (Fig. 2). IgG-IgA2-containing CIC were elevated in only 2 of 30 (7\%) patients, both of whom also had IgG-IgAl CIC. There were no significant correlations between levels of IgG-IgA 1 CIC and either serum creatinine concentrations or magnitudes of protein-

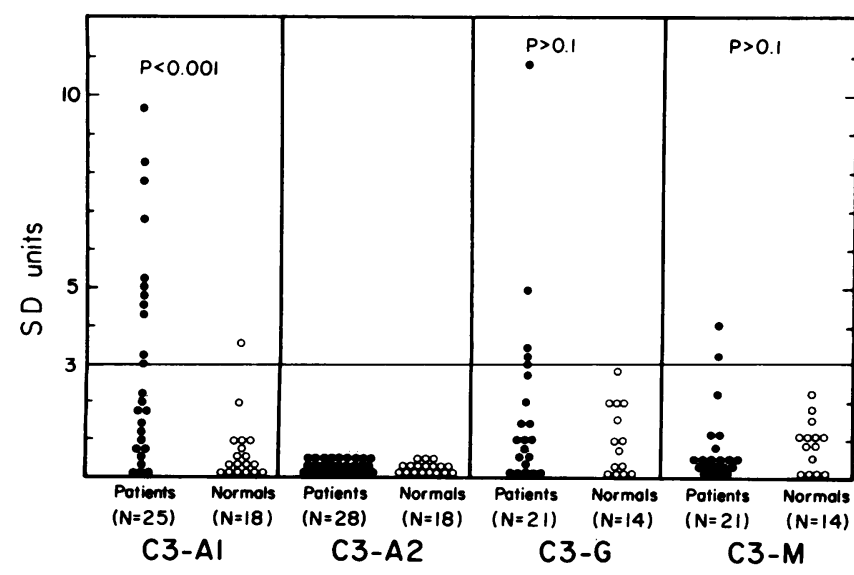

Figure 1. Levels of CIC containing IgA1, IgA2, IgG, or IgM in IgA NP patients and normal controls as determined by the anti-C 3 assay. Values are expressed in standard deviation (SD) units as described in Methods.

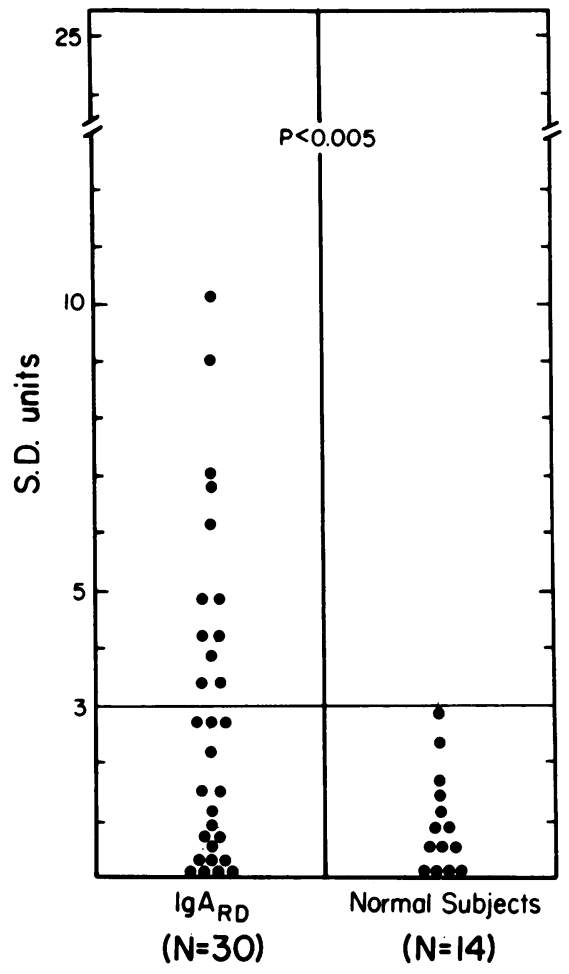

Figure 2. Levels of CIC containing both IgAl and IgG in IgA NP patients and normal subjects as determined by the RIA. Values are expressed as in Fig. 1.

uria or hematuria. Increased levels of CIC containing both IgM and IgAl were detected in $16 \%(5 / 30)$ of the patients studied; all five patients also had increased levels of IgG-IgAl CIC. However, no correlation was observed between levels of CIC containing IgA1 and either IgG or IgM. In addition, none of these patients had evidence of CIC containing both IgA2 and IgM.

Sedimentation characteristics of IgA-containing CIC. Sucrose gradient ultracentrifugation analyses of redissolved serum PEG precipitates obtained from four patients with high levels of CIC containing IgA 1-C3 revealed that these CIC sedimented over a wide range of sizes, from $11 \mathrm{~S}$ to $>19 \mathrm{~S}$ (Fig. 3). CIC containing IgG and IgA1 were predominantly of intermediate (13-19S) and occasionally of large (>19S) size (Fig. 4). Sucrose gradient analyses of affinity-isolated $\mathrm{CIC}$ containing $\mathrm{C} 3$ and $\operatorname{IgA}$ revealed the presence of a significant proportion of 7S IgA and of polymeric (or aggregated) IgA upon acid dissociation (Fig. 5). The same type of analyses performed on isolated CIC containing both $\operatorname{IgA}$ and $\operatorname{IgG}$ indicated that the IgA component of these CIC was predominantly monomeric (Fig. 6).

Serum levels of $R F$. The presence of IgA1-IgG CIC in approximately one half of IgA NP patients raised the possibility that IgA anti-IgG antibodies might be a constituent of these complexes. In order to examine this possibility, RF levels were assayed in the serum of these patients. Serum levels of IgA RF were elevated in $28 \%(7 / 25)$ and $32 \%(8 / 25)$ of the patients as detected by RIA and ELISA PAP, respectively (Fig. 7). IgM RF levels were within the normal range by both procedures with the exception of one patient who had increased serum levels of both IgA RF and IgM RF by RIA. IgG RF levels, as assessed by the ELISA-PAP procedure, were increased in only $14 \%$ of the 


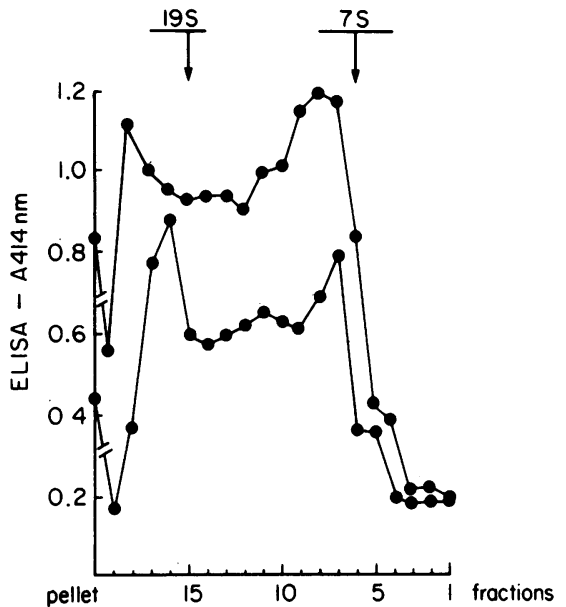

Figure 3. Sedimentation properties of IgA1-C3 CIC. Redissolved PEG precipitates from the sera of two IgA NP patients were ultracentrifuged on linear sucrose gradients. Collected fractions were assayed by the anti-C3 method for IgA 1-C3 CIC. Arrows indicate the position of IgM (19S) and IgG (7S) standards.

patients. IgA1 was the exclusive subclass of IgA RF detected in all of five sera examined. In addition, the IgA 1 RF detected in these sera was reactive with the Fc portion of human IgG as determined by RIA using solid-phase Fc fragments and $F\left(a^{\prime}\right)_{2}$ of human IgG. There was no significant correlation between serum IgA RF levels and levels of CIC containing C3 and IgA. Borderline statistical significance was observed between serum IgA RF levels and levels of CIC containing both IgG and IgAI $(r=0.38 ; P=0.06)$. It should be emphasized that six of seven (85\%) patients with elevated IgA RF had CIC containing IgAI and IgG (Table I).

Sedimentation characteristics of IgA RF present in serum and in isolated $\mathrm{CIC}$. Sucrose gradient ultracentrifugation analyses of serum samples from four patients with high levels of IgAl RF showed that the RF sedimented in fractions corresponding to monomeric as well as polymeric IgA (Fig. 8). Parallel analyses performed at acid $\mathrm{pH}$ yielded similar results. Thus a portion of the IgA RF detected was truly polymeric and was not composed of aggregates. Analogous experiments performed on isolated IgG-IgA CIC from selected patients showed that part of the IgA

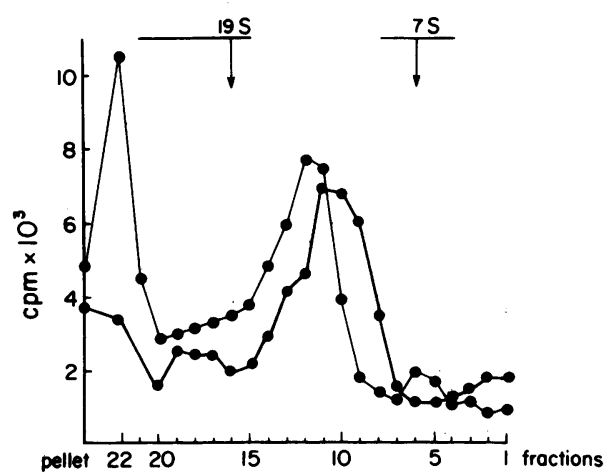

Figure 4. Sedimentation profile of CIC containing both IgG and IgAI. Redissolved PEG precipitates from the sera of two IgA NP patients were ultracentrifuged on linear sucrose gradients. Collected fractions were analyzed for IgG-IgAI CIC by the RIA procedure.

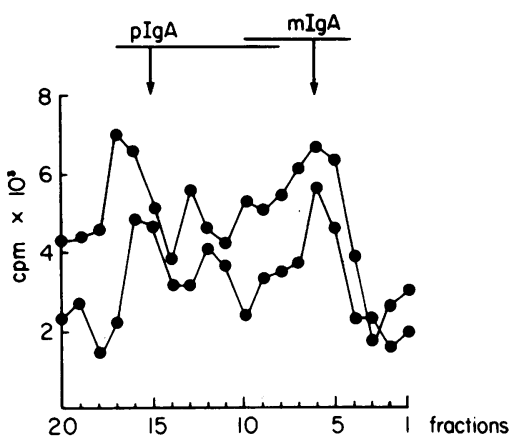

Figure 5. Sedimentation profile of IgA present in CIC containing C3 and IgA. Redissolved PEG precipitates from the sera from two patients with IgA NP were incubated with anti-C3-conjugated Sepharose 4B. Bound material was eluted with acid buffer. After ultracentrifugation on linear sucrose gradients in acid buffer, the collected fractions were neutralized, diluted i:50, and subsequently assayed for IgA content by solid-phase RIA. Arrows indicate the position of the standards. These consisted of purified monomeric IgA (mIgA) and polymeric IgA (pIgA) paraproteins.

exhibited RF activity upon dissociation at acid pH. IgA RF was found predominantly in fractions corresponding to polymeric IgA (Fig. 9).

\section{Discussion}

Although IgA CIC levels have been correlated with episodes of nephritis and with recurrence of mesangial IgA nephropathy after renal transplantation (15), others have shown that IgA CIC persist irrespective of clinical nephritis $(20,21,25,36)$. This discrepancy may be due to the use of assays that detect different types of CIC or to utilization of different procedures for the detection of IgA in $\mathrm{CIC}$. The assays described here offer sensitive and specific methods for estimation of CIC containing IgA and $\mathrm{C} 3$ and of CIC of mixed immunoglobulin classes regardless of the presence of complement components. Initial experiments

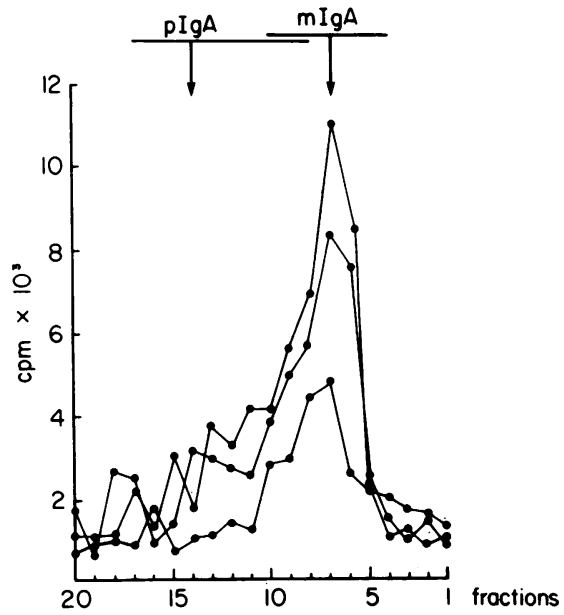

Figure 6. Sedimentation profile of IgA present in CIC containing both IgG and IgA. Redissolved PEG precipitates from three patients with IgA NP were incubated with anti-IgG conjugated Sepharose 4B. Bound material was eluted, concentrated, and analyzed for IgA content as in Fig. 5. 


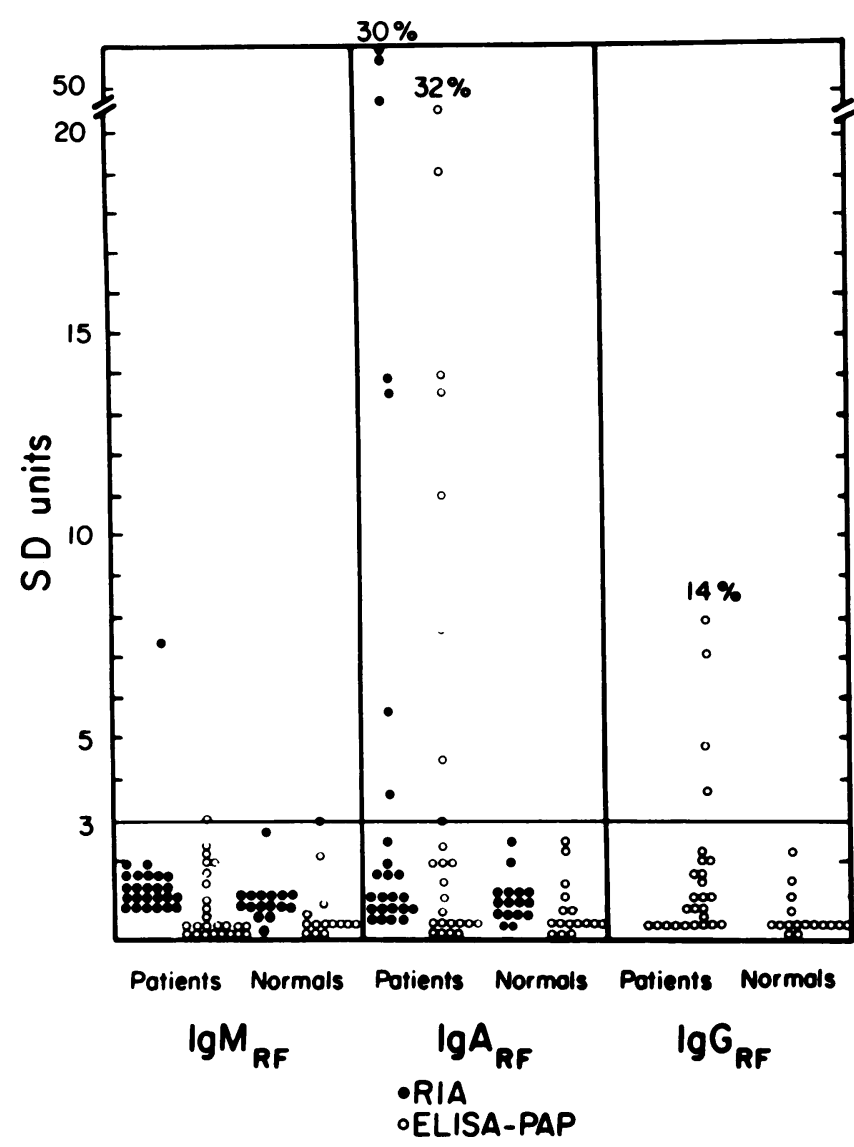

Figure 7. Serum levels of IgM-, IgA-, and IgG-RF in patients with IgA NP and in healthy volunteers as determined by solid-phase RIA (๑) and ELISA-PAP (O). Values are given in standard deviation (SD) units as in Figs. 1 and 2.

performed in our laboratory established that PEG precipitation was preferable to the use of whole sera because it provided more discriminative results in both $\mathrm{CIC}$ assays. Our experience is in agreement with Kauffman et al. (41), who have demonstrated the efficacy of PEG precipitation for the detection of IgA CIC. In this regard, pretreatment with PEG probably minimizes the competitive effects of free $\mathrm{C} 3$ and immunoglobulin present in whole serum.

In agreement with previous reports (14-26), we found that approximately half of the patients with IgA NP had increased levels of IgA-CIC detectable in the $\mathrm{F}\left(\mathrm{ab}^{\prime}\right)_{2}$ anti-C3 assay. In addition, the IgA component of the anti-C3-reactive material was confined exclusively to the IgA 1 subclass. This finding is at vari-

Table I. Comparison between Serum Levels of IgA RF and IgAl-containing CIC

\begin{tabular}{llllll}
\hline & \multicolumn{2}{l}{ IgG-IgAI CIC } & & \multicolumn{2}{l}{ C3-IgAl CIC } \\
\cline { 2 - 3 } \cline { 5 - 6 } \cline { 5 - 6 } & $\begin{array}{l}\text { Positive } \\
(10 / 25)\end{array}$ & $\begin{array}{l}\text { Negative } \\
(15 / 25)\end{array}$ & & $\begin{array}{l}\text { Positive } \\
(11 / 25)\end{array}$ & $\begin{array}{l}\text { Negative } \\
(14 / 25)\end{array}$ \\
\hline IgA RF-positive $(7 / 25)$ & $6^{*}$ & 1 & & $3 \ddagger$ & 4 \\
IgA RF-negative (18/25) & 4 & 14 & & 8 & 10 \\
\hline
\end{tabular}

$* P=0.06$

$\ddagger P=0.53$.

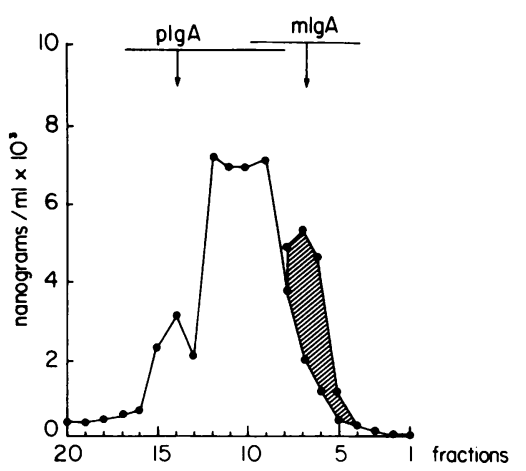

Figure 8. Sedimentation profile of serum IgA RF in a patient with IgA $\mathrm{NP}$ as determined by sucrose gradient ultracentrifugation. Collected fractions were assayed by solid-phase RIA for IgA RF activity. The shaded area indicates the difference in IgA RF activity between fractions treated and fractions not treated with mouse monoclonal antiIgA antibody as cross-linking agent. Arrows indicate the position of polymeric IgA (pIgA) and monomeric IgA (mIgA) RF paraprotein standards.

ance with an earlier report (52) in which both $\operatorname{IgA} 1$ and $\operatorname{IgA} 2$ were detected in conglutinin-reactive CIC material. The reason for this discrepancy may be related to the use of different CIC assays and/or anti-IgA subclass reagents. A substantial proportion of our patients had increased levels of CIC that contained both IgG and IgA 1 , whereas those that contained IgM and IgA I were elevated in only a few patients. $\operatorname{IgA} 2$ was rarely detected in association with IgG or IgM thus providing further evidence that this subclass is not a common component of IgA-CIC detected in IgA NP patients.

The presence of $\mathrm{IgG}$ in IgA 1-containing $\mathrm{CIC}$ may explain the unexpected detection of IgA CIC by the Clq-binding test in $43 \%$ of IgA NP patients (11). This procedure is often used for the demonstration of CIC containing $\operatorname{IgG}$ or $\operatorname{IgM}(53,54)$ but not IgA, which does not appear to activate complement by the classical pathway (55). Thus, it is likely that the Clq-reactive material may have been due to the presence of $\mathrm{IgG}$ and $\mathrm{IgA}$ in the same CIC matrix.

Our results revealed considerable heterogeneity of $\mathrm{CIC}$ in IgA NP patients. First, IgA1-IgG CIC did not correlate with CIC containing either IgA1-C3 or IgG-C3. Secondly, sucrose gradient ultracentrifugation analyses demonstrated that IgA1-IgG CIC had different sedimentation profiles from those containing IgA 1 and C3. Thirdly, isolated IgA CIC contained polymeric and substantial amounts of monomeric IgA after acid dissociation. The predominance of IgA monomers and the rarity of IgA2 suggest that $\mathrm{CIC}$ containing IgA 1 are not likely to be formed at mucosal sites (56).

The presence of $\mathrm{CIC}$ that contain IgAl and IgG raises several questions as to the nature and the possible mechanisms of their formation. First, IgG and IgAl might be bound to the same antigen(s) through either identical or different epitopes. Second, the IgG and IgAl components may specifically interact with each other. In this regard, we demonstrated the presence of IgA $\mathrm{RF}$ in the sera of approximately one third of IgA NP patients in the absence of increased IgM RF. IgA RF was confined exclusively to the IgAl subclass and occurred in both monomeric and polymeric forms. It is likely that part of the IgA 1 RF activity detected may be associated with autologous IgG and as such contribute to the population of CIC that contain IgA1. This 

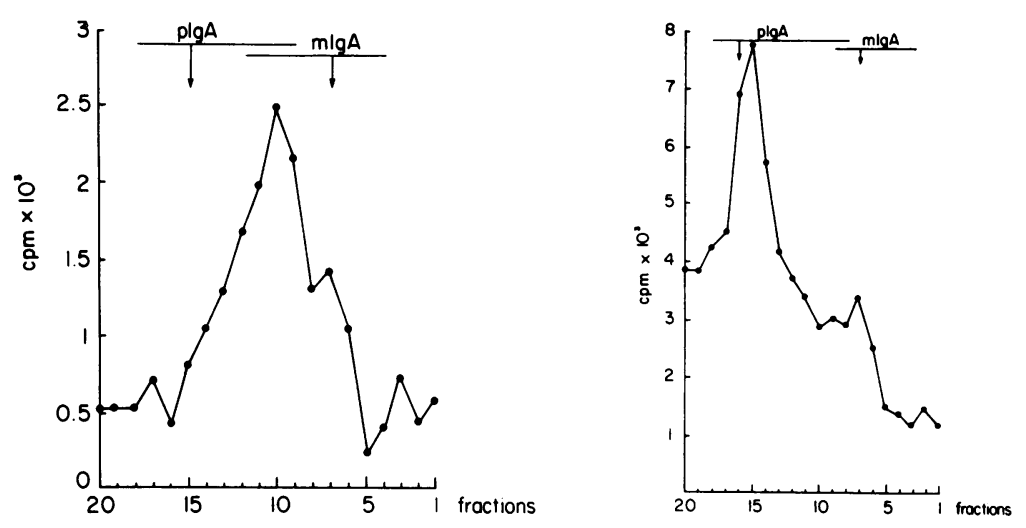

Figure 9. Sedimentation profile of IgA RF present in isolated CIC from two patients with IgA NP. CIC containing IgG and IgA were isolated by affinity chromatography on anti-IgG Sepharose 4B. After acid elution and sucrose gradient ultracentrifugation at acid $\mathrm{pH}$, fractions containing polymeric (pIgA) or monomeric IgA (mIgA) were neutralized and assayed for IgA RF activity by solid-phase RIA as in Fig. 8. view is supported by the demonstration of increased levels of IgA1-IgG CIC in most patients with elevated serum levels of IgA RF, and by the detection of IgA RF in isolated IgA-IgG CIC. The observation that IgA1-IgG CIC could occur in the absence of detectable serum IgA RF raises the possibility of additional recognition systems such as idiotype-anti-idiotype interactions or IgG auto-anti-IgA1 antibodies. On the other hand, it may also reflect in vivo competitive binding of IgA1 RF molecules to an excess of endogenous IgG which could interfere with the detection of IgA RF in some patients.

The selective increase of IgA RF observed in our study has not so far been reported in patients with IgA NP or, to our knowledge, in other diseases. This observation has several implications. It supports the contention that IgA RF production and IgM RF production are controlled by distinct regulatory mechanisms (57). Thus, selective production of IgAl RF may reflect altered immunoregulatory function in this disease. Although the mechanisms of IgA RF induction are unknown, they could be analogous to those implicated for IgM RF. IgA RF may represent a secondary response to immunologically complexed IgG as recently demonstrated with regard to IgM RF production in mice $(58,59)$ and humans $(42)$. Because $C 3$ and even IgM co-deposit with IgA in glomeruli more frequently than IgG (34), the possibility that IgA anti-C3 or IgA anti-IgM autoantibodies occur in the circulation of these patients was considered. However, we have been unable to demonstrate free $\operatorname{IgA}$ antibodies to either C3 or IgM in sera of selected patients (unpublished results).

There is a voluminous literature, often contradictory, concerning abnormalities of immunoregulatory mechanisms of the IgA system observed in IgA NP patients (for review, see Clarkson et al. [7] and Lomax-Smith et al. [60]). These include increased serum levels of $\operatorname{IgA}$, often elevated numbers of IgA-bearing B cells in the peripheral blood, and increased IgA production in vitro, possibly owing to the decrease of $\mathrm{T}$ suppressor cells and increase in $\mathrm{T}$ helper cells. Our study provides further evidence for altered immunoregulation of IgA synthesis by showing the selective presence of IgA $1 \mathrm{CIC}$ and IgA1 RF in these patients. This IgA1 subclass restriction is remarkable in view of the fact that most IgA antibody responses to a variety of antigens involve both subclasses $(34,61)$.

Levels of IgA1-C3 CIC correlated with the magnitude of proteinuria but not with the degree of renal insufficiency or the magnitude of hematuria. However, neither IgA1-IgG CIC nor IgA RF correlated with any of these indices of glomerular damage. This suggests that the different types of IgA1 CIC may have different roles. Nevertheless, the clinical significance of our observations, their relationship to the development or progression of renal insufficiency in IgA NP, and their role in the pathogenesis of the disease must remain speculative at this time.

In conclusion, our immunochemical data taken together with previous immunohistochemical findings $(18,31-34)$ suggest a dominant role of the IgAl subclass in the pathogenesis of IgA NP. The lack of detectable alterations of the IgA compartment of mucosa-associated tissues (62) and the physical properties of the IgA1 associated with IgA CIC as well as IgA RF suggest a nonmucosal origin for a large proportion of these components.

\section{Acknowledgments}

We thank Drs. John Kearney, Jiri Radl, and J. Haaijman for providing the monoclonal antibody reagents used in this study. The technical assistance of Ms. Shirley Prince, Ms. Rose Kulhavy, and Ms. Marilyn Spotswood is gratefully appreciated. We are particularly indebted to Drs. R. W. Baehler, C. E. Cooley, J. J. Curtis, E. C. Kohaut, J. W. Jackson, R. G. Luke, W. L. McGuffin, Jr., R. G. McMorrow, C. W. Old, J. R. Reed, S. J. Rostand, E. A. Rutsky, F. X. Schloeder, T. K. Taylor, D. Tharpe, B. Waldo, and J. Work for allowing us to study their patients.

This work was supported by grants AI-18745, AI-10854, AM-28357, and AM-03555 from the National Institutes of Health and the Veterans Administration Research Program.

\section{References}

1. D’Amico, G., E. Imbasciati, G. B. Barbiano di Belgiojoso, S. Bertoli, G. Fogazzi, G. Ferrario, G. Fellin, A. Ragni, G. Colasanti, L. Minetti, and C. Ponticelli. 1985. Idiopathic IgA mesangial nephropathy: clinical and histological study of 374 patients. Medicine (Baltimore). 64:49-60.

2. Berger, J., and N. Hinglais. 1968. Les depôts intercapillaires d'IgAIgG. J. Urol. Nephrol. (Paris). 784:694-695.

3. Baart de la Faille-Kuyper, E. H., L. Kater, C. J. Kooiker, and E. J. Dorhout Mees. 1973. IgA deposits in cutaneous blood vessel walls and mesangium in Henoch-Schoenlein syndrome. Lancet. i:892-894.

4. Callard, P., G. Feldmann, D. Prandi, M. F. Belair, C. Mandet, Y. Weiss, P. Druet, J. P. Benhamou, and J. Bariety. 1975. Immune complex type glomerulonephritis in cirrhosis of the liver. Am. J. Pathol. 80:329340.

5. Nochy, D., P. Callard, B. Bellon, J. Bariety, and P. Druet. 1976. Association of overt glomerulonephritis and liver disease: A study of 34 patients. Clin. Nephrol. 6:422-427.

6. Giangiacomo, J., and C. C. Tsia. 1977. Dermal and glomerular deposition of IgA in anaphylactoid purpura. Am. J. Dis. Child. 131:981987.

7. Clarkson, A. R., A. E. Seymour, A. J. Thompson, W. D. G. Haynes, 
Y-L. Chan, and B. Jackson. 1977. IgA nephropathy: a syndrome of uniform morphology, diverse clinical features and uncertain prognosis. Clin. Nephrol. 8:459-471.

8. Nakamoto, Y., Y. Asano, K. Dohi, M. Fujioka, H. lida, H. Kida, Y. Kibe, K. N. Hattori, and J. Takeuchi. 1978. Primary IgA glomerulonephritis and Schoenlein-Henoch purpura nephritis: Clinicopathological and immunohistochemical characteristics. Q. J. Med. 47:495-516.

9. Berger, J., H. Yaneva, and B. Nabarra. 1978. Glomerular changes in patients with cirrhosis of the liver. Adv. Nephrol. 7:3-14.

10. Katz, A., R. F. Dyck, and R. A. Bear. 1979. Celiac disease associated with immune complex glomerulonephritis. Clin. Nephrol. 11: 39-46.

11. Woodroffe, A. J., A. J. Gormly, P. E. McKenzie, A. M. Wooton, A. J. Thompson, A. E. Seymour, and A. R. Clarkson. 1980. Immunologic studies in IgA nephropathy. Kidney Int. 18:366-374.

12. Emancipator, S. N., G. R. Gallo, and M. E. Lamm. 1981. IgA nephropathy: Clinical, morphologic, and immunologic features. Surg. Pathol. Q. Index. 3:179-190.

13. Berger, J., H. Yaneva, B. Nabarra, and C. Babanel. 1975. Recurrence of mesangial deposition of IgA after renal transplantation. Kidney Int. 7:232-241.

14. Stachura, I., G. Singh, and T. L. Whiteside. 1981. Immune abnormalities in IgA nephropathy (Berger's disease). Clin. Immunol. Immunopathol. 20:373-388.

15. Lesavre, P. H., M. Digeon, and J. F. Bach. 1982. Analysis of circulating IgA and detection of immune complexes in primary IgA nephropathy. Clin. Exp. Immunol. 48:61-69.

16. Coppo, R., B. Basolo, G. Martina, C. Rollino, M. De Marchi, F. Giacchino, G. Mazzucco, M. Messina, and G. Piccoli. 1982. Circulating immune complexes containing IgA, IgG and IgM in patients with primary IgA nephropathy and with Henoch-Schoenlein nephritis. Correlation with clinical and histologic signs of activity. Clin. Nephrol. 18:230-239.

17. Doi, T., K. Kanatsu, H. Sekita, H. Yoshida, H. Nagai, and Y. Hamashima. 1982. Circulating immune complexes of IgG, IgA and IgM classes in various glomerular diseases. Nephron. 32:335-341.

18. Hall, R. P., I. Stachura, J. Cason, T. L. Whiteside, and T. J. Lawley. 1983. IgA containing circulating immune complexes in patients with IgA nephropathy. Am. J. Med. 74:56-63.

19. Valentijn, R. M., R. H. Kauffmann, G. Brutel de la Riviere, M. R. Daha, and L. A. van Es. 1983. Presence of circulating macromolecular IgA in patients with hematuria due to primary IgA nephropathy. Am. J. Med. 74:375-381.

20. Egido, J., J. Sancho, F. Rivera, and L. Hernando. 1984. The role of IgA and IgG immune complexes in IgA nephropathy. Nephron. 36: 52-59.

21. Levinsky, R. J., and T. M. Barratt. 1979. IgA immune complexes in Henoch-Schoenlein purpura. Lancet. ii:1100-1103.

22. Kauffman, R. H., W. A. Herrman, C. J. L. M. Meyer, M. Daha, and L. A. van Es. 1980. Circulating IgA immune complexes in HenochSchoenlein purpura. Am. J. Med. 69:859-866.

23. Hall, R. P., T. J. Lawley, J. A. Heck, and S. I. Katz. 1980. IgA containing circulating immune complexes in dermatitis herpetiformis, Henoch-Schoenlein purpura, systemic lupus erythematosus and other diseases. Clin. Exp. Immunol. 40:431-437.

24. Zone, J. J., B. A. LaSalle, and T. T. Provost. 1980. Circulating immune complexes of IgA type in dermatitis herpetiformis. J. Invest. Dermatol. 75:152-155.

25. Penner, E., B. Albini, and F. Milgrom. 1978. Detection of circulating immune complexes in alcoholic disease. Clin. Exp. Immunol. 34:28-31.

26. Coppo, R., S. Arico, G. Piccoli, B. Basolo, D. Roccatello, A. Amore, M. Tabone, M. de la Pierre, A. Sessa, D. L. Delacroix, and J. P. Vaerman. 1985. Presence and origin of IgA1- and IgA2-containing circulating immune complexes in chronic alcoholic liver diseases with and without glomerulonephritis. Clin. Immunol. Immunopathol. 35:1-8.

27. Rifai, A., P. A. Small, P. O. Teague, and E. M. Ayoub. 1979. Experimental IgA nephropathy. J. Exp. Med. 150:1161-1173.
28. Emancipator, S. N., G. R. Gallo, and M. E. Lamm. 1983. Experimental IgA nephropathy induced by oral immunization. J. Exp. Med. 157:572-582.

29. Issacs, K., F. Miller, and B. Lane. 1981. Experimental model for IgA nephropathy. Clin. Immunol. Immunopathol. 20:419-426.

30. Andre, C., F. C. Berthoux, F. Andre, J. Gillon, C. Genin, and J. C. Sabatier. 1980. Prevalence of IgA2 deposits in IgA nephropathies. N. Engl. J. Med. 303:1343-1346.

31. Conley, M. E., M. D. Cooper, and A. F. Michael. 1980. Selective deposition of immunoglobulin Al in immunoglobulin A nephropathy, anaphylactoid purpura nephritis and systemic lupus erythematosus. $J$. Clin. Invest. 66:1432-1436.

32. Tomino, Y., M. Endoh, Y. Nomoto, and H. Sakai. 1981. Immunoglobulin Al in IgA nephropathy. N. Engl. J. Med. 305:1159-1160.

33. Valentijn, R. M., J. Radl, J. J. Haaijman, B. J. Vermeer, J. J. Veening, R. H. Kauffmann, M. R. Daha, and L. A. van Es. 1984. Circulating and mesangial secretory component-binding IgA1 in primary IgA nephropathy. Kidney Int. 26:760-766.

34. Russell, M. W., J. Mestecky, B. A. Julian, and J. H. Galla. 1986. IgA-associated renal diseases. Antibodies to environmental antigens in sera and deposition of immunoglobulins and antigens in glomeruli. $J$. Clin. Immunol. 6:74-86.

35. Dobrin, R. S., F. E. Knudson, and A. F. Michael. 1975. The secretory immune system and renal disease. Clin. Exp. Immunol. 21: 318-328.

36. Whitworth, J. A., S. Leibowitz, M. C. Kennedy, J. S. Cameron, and J. C. Chantler. 1976. IgA and glomerular disease. Clin. Nephrol. 5: 33-36.

37. Thomas, H. C., D. DeVilliers, B. Potter, H. Hodgson, S. Jain, D. P. Jewell, and S. Sherlock. 1978. Immune complexes in acute and chronic liver disease. Clin. Exp. Immunol. 31:150-157.

38. Abrass, C. K., W. A. Border, and G. Hepner. 1982. Non-specificity of circulating immune complexes in patients with acute and chronic liver disease. Clin. Exp. Immunol. 40:292-301.

39. Clarkson, A. R., A. J. Woodroffe, K. M. Bannister, J. D. LomaxSmith, and I. Aarons. 1984. The syndrome of IgA nephropathy. Clin. Nephrol. 21:7-14.

40. Galla, J. H., E. C. Kohaut, R. Alexander, and J. Mestecky. 1984. Racial difference in the prevalence of IgA-associated nephropathies. Lancet. ii:522.

41. Kauffman, R. H., L. A. van Es, and M. R. Daha. 1981. Specific detection of IgA immune complexes. J. Immunol. Methods. 40:117129.

42. Tarkowski, A., C. Czerkinsky, and L. A. Nilsson. 1985. Simultaneous induction of rheumatoid factor- and antigen-specific antibody secreting cells during the secondary immune response in man. Clin. Exp. Immunol. 61:379-387.

43. Pereira, A. B., A. N. Theophilopoulos, and F. J. Dixon. 1980. Detection and partial characterization of circulating immune complexes with solid-phase anti-C3. J. Immunol. 125:763-772.

44. Kutteh, W. H., S. J. Prince, J. O. Phillips, J. G. Spenney, and J. Mestecky. 1982. Properties of immunoglobulin A in serum of individuals with liver diseases and in hepatic bile. Gastroenterology. 82:184-193.

45. Mestecky, J., W. J. Hammack, R. Kulhavy, G. P. Wright, and M. Tomana. 1977. Properties of IgA myeloma proteins isolated from sera of patients with the hyperviscosity syndrome. J. Lab. Clin. Med. 89:919-927.

46. Crago, S. S., W. H. Kutteh, I. Moro, M. R. Allansmith, J. Radl, J. J. Haaijman, and J. Mestecky. 1984. Distribution of IgA1-, IgA2- and $\mathrm{J}$ chain-containing cells in human tissues. J. Immunol. 132:16-18.

47. Kubagawa, H., M. Mayumi, J. F. Kearney, and M. D. Cooper. 1982. Immunoglobulin $V_{H}$ determinants defined by monoclonal antibodies. J. Exp. Med. 156:1010-1021.

48. Marchalonis, J. J. 1969. An enzymic method for the trace iodination of immunoglobulins and other proteins. Biochem. J. 113:299-305.

49. Koopman, W. J., R. E. Schrohenloher, and A. Solomon. 1982. A quantitative assay for IgA rheumatoid factor. J. Immunol. Methods. 50:89-98. 
50. Koopman, W. J., and R. E. Schrohenloher. 1980. A sensitive radioimmunoassay for quantitation of IgM rheumatoid factor. Arthritis Rheum. 23:302-308.

51. Schrohenloher, R. E., W. J. Koopman, Z. Moldoveanu, and A. Solomon. 1985. Activity of rheumatoid factors of different molecular sizes; comparison of autologous monomeric and polymeric monoclonal IgA rheumatoid factors. J. Immunol. 134:1469-1474.

52. Coppo, R., B. Basolo, G. Piccoli, G. Mazzucco, M. R. Bulzomi, D. Roccatello, M. De Marchi, A. O. Carbonara, and G. Barbiano Di Belgiojoso. 1984. IgA1 and IgA2 immune complexes in primary IgA nephropathy and Henoch-Schoenlein nephritis. Clin. Exp. Immunol. 57:583-590.

53. Nydegger, U. E., P. H. Lambert, H. Gerber, and P. H. Miescher. 1974. Circulating immune complexes in the serum in systemic lupus erythematosus and in carriers of hepatitis B antigen. J. Clin. Invest. 54: 297-309.

54. Lurhuman, A. Z., C. L. Cambiaso, P. L. Masson, and J. F. Heremans. 1976. Detection of circulating antigen-antibody complexes by their inhibitory effect on the agglutination of IgG-coated particles by rheumatoid factor or Clq. Clin. Exp. Immunol. 25:212.

55. Boackle, R. J., K. M. Pruitt, and J. Mestecky. 1974. The interactions of human complement with interfacially aggregated preparations of human secretory IgA. Immunochemistry. 11:543-548.
56. Katz, A., M. M. Newkirk, and M. H. Klein. 1984. Circulating and mesangial IgA in IgA nephropathy. In IgA Mesangial Nephropathy. D’Amico, G., Minetti, L., Ponticelli, C. editors. S. Karger, Basel. 74-79.

57. Koopman, W. J., R. E. Schrohenloher, S. S. Crago, D. M. Spalding, and J. Mestecky. 1985. IgA rheumatoid factor synthesis by dissociated synovial cells. Characterization and relationship to IgM rheumatoid factor synthesis. Arthritis Rheum. 28:1219-1227.

58. Coulie, P. G., and J. Van Snick. 1985. Rheumatoid factor (RF) production during anamnestic immune responses in the mouse III. Activation of RF precursor cells is induced by their interaction with immune complexes and carrier-specific helper T cells. J. Exp. Med. 161:88-97.

59. Nemazee, D. A. 1985. Immune complexes can trigger specific $T$ cell dependent, autoanti-IgG antibody production in mice. J. Exp. Med. 161:252-256.

60. Lomax-Smith, J. D., A. J. Woodroffe, A. R. Clarkson, and A. E. Seymour. 1985. IgA nephropathy-accumulated experience and current concepts. Pathology. 17:219-224.

61. Conley, M. E., and D. E. Briles. 1984. Lack of IgA subclass restriction in antibody response to phosphorylcholine, $\beta$-lactoglobulin and tetanus toxoid. Immunology. 53:419-426.

62. Westberg, N. G., K. Baklien, B. Schmekel, R. Gillberg, and P. Brandtzaeg. 1983. Quantitation of immunoglobulin-producing cells in small intestinal mucosa of patients with IgA nephropathy. Clin. Immunol. Immunopathol. 26:442-445. 